\title{
La Preinversión en la creación de empresas. Una forma de concretar ideas prometedoras y tomar decisiones exitosas.
}

JUAN JOSE MIRANDA MIRANDA ${ }^{27}$

Al observar las precarias tasas de crecimiento que reportan los países emergentes especialmente los de América Latina y, compararlas con los índices de crecimiento vegetativo de su población encontramos serios motivos de alarma con respecto a la capacidad de estas economías para facilitar empleo a buena parte de su población en edad laboral. En efecto, los índices de desempleo, subempleo y empleo disfrazado en la región están por niveles entre el $12 \%$ y el $25 \%$. Solamente con economías dinámicas creciendo por lo menos al un $5 \%$ anual se puede ir reduciendo esta humillante tasa.

Pero la única posibilidad de acelerar el ritmo de crecimiento de la región y por ende de las economías nacionales, y de crear puestos de trabajo suficientes, reside en la gestación de nuevos negocios o la ampliación de los existentes. Es claro que el sector público no tiene suficiente capacidad financiera ni necesidad de emplear más gente, antes por el contrario, en pos de mayor eficiencia debe disminuir sus nóminas. Por otro lado, las grandes empresas no están en plan de expansión de inversiones, sino de consolidar los procesos de ajuste estratégico, reestructuración de sus finanzas y modernización tecnológica que les permita ser competitivas en un mercado marcadamente globalizado. Por lo tanto, en América Latina se deberán activar y emplear a fondo todos los mecanismos disponibles que estimulen la creación, financiación y puesta en marcha de nuevas unidades empresariales y la consolidación y ampliación de las existentes. Por esta razón no nos cansaremos de afirmar que "la inversión nueva es el motor de la economía".

Son muchas las ideas prometedoras que en el interior de las empresas públicas y privadas, se pierden, ya porque no encuentran un ámbito adecuado para exponerlas, ya porque no existen canales idóneos de comunicación, porque el funcionario o empleado no recibe estímulo para mejorar su labor, o simplemente, porque se está tan inmerso en la rutina del día que no permite a directivos y subalternos observar las oportunidades y prever los peligros propios de un mundo dinámico que cada día exige mayor eficiencia en quienes prestan servicios o producen bienes. De ahí la necesidad de crear e impulsar al interior de las empresas públicas y privadas, para que no se pierdan las buenas ideas, ambientes propicios a la "estructuración de proyectos"28.

\footnotetext{
${ }^{27}$ Economista, Universidad Externado de Colombia. Especialista en Análisis de Proyectos, ESAP-OEA. Autor de varios libros en Proyectos. Consultor y catedrático.

${ }^{28}$ Los llamados "bancos de proyectos" se constituyen en la herramienta idónea para canalizar esta capacidad de creación e innovación al interior de las organizaciones.
} 
Sin duda la incursión de la nueva tecnología y el desarrollo de las comunicaciones han determinado cambios en los paradigmas, a más de la composición de los productos. En los últimos años, por ejemplo, existe un superávit mundial de productos agrícolas ${ }^{29}$ determinado por la expansión de la producción rural en el mundo en desarrollo y la aparición de nuevas tecnologías que como los transgénicos incrementan su rendimiento. Los países emergentes y en particular los de América Latina vienen financiando su desarrollo o sea pagando las importaciones con el producto de las exportaciones masivas de alimentos y materias primas. El observar la caída de los precios internacionales por mayores niveles de oferta, nos mueve a buscar modelos y estrategias alternativas que incorporen mayor valor agregado a través de nuevas empresas transformadoras.

Parece que la composición y los costos que soportan los nuevos esquemas empresariales tiene una notable participación en aquellos productos o servicios que incorporan en forma intensiva "conocimiento e información" y menores participaciones a los materiales y la mano de obra de baja calificación. El mundo evoluciona menos hacia el uso intensivo de mano de obra, de materiales, de capital o de energía, y sí hacia el uso intensivo de conocimientos. Al decir de Peter Drucker30 "una compañía que opere hoy a un costo de trabajo manual superior al $15 \%$ queda fuera del mercado".

En el nuevo siglo la capacidad de trasmitir ideas e información rápidamente y a bajo costo será la norma para mejorar la productividad. Hoy sabemos que el conocimiento y la información constituyen el origen de la riqueza. Si lo aplicamos a las tareas que ya sabemos ejecutar, se denominará "productividad"; si lo utilizamos en tareas nuevas y diferentes, se llamará "innovación". Sólo el conocimiento y la información nos permiten alcanzar esos dos objetivos. Las utilidades y la rentabilidad, cada vez más, estarán ligadas al incremento de la productividad y la búsqueda de procesos innovadores, lo contrario evidenciará una tendencia hacia la destrucción de valor. La productividad es la verdadera ventaja competitiva, las ventajas comparativas poco a poco se van extinguiendo.

El desarrollo asombroso y los avances constantes de la tecnología de la comunicación y la información han compactado la geografía mundial, aglomerando los mercados de toda gama de bienes y servicios, planteando un permanente desafío a gobiernos y empresarios. En efecto, la geopolítica mundial tiene una inédita reconfiguración al diseñar y apropiar procesos de integración supranacionales determinando la homogeneidad de políticas, procedimientos y reglas de operación de los conglomerados económicos. El resultado se manifiesta en el surgimiento de nuevos y más amplios estadios donde se implantan estrategias globales que seducen y cautivan a grupos importantes de la población mundial. Por lo tanto, es preciso impulsar una vocación exportadora vigorosa y sostenida, asignándole un lugar importante en la estrategia de desarrollo. De hecho, los paradigmas propios de la "nueva economía" y la necesaria visión internacional de las decisiones que se toman en medio del ámbito de la globalización cada vez más competitivo y cargado de incertidumbre, determinan la necesidad de una visión amplia y global, más allá de las fronteras.

${ }^{29} \mathrm{~A}$ pesar que en el mundo emergente muchos de sus habitantes se mueren de hambre.

${ }^{30}$ Peter Drucker, Gerencia para el Futuro. 
Las empresas del futuro tendrán que ser transnacionales, las fronteras no será impedimento válido para su crecimiento. Estarán donde se requiera de sus productos y el suministro de recursos se acomode mejor a su competitividad. De ahí surge la necesidad de impregnar también en el nuevo espíritu empresarial la vocación exportadora.

Para que el espíritu empresarial produzca resultados concretos, no basta con tener buenas ideas, se precisa saberlas exponer y apoyarlas con argumentos válidos, por eso la necesidad de aprender a "estructurar proyectos" que den salida a buenos y confiables "planes de negocios". Tenemos el testimonio de muchos planes de negocios que no son otra cosa que un listado de buenas intenciones sin respaldo ni argumento alguno, que denuncia el desconocimiento del sector y las reglas de juego del mercado al cual pretenden apuntar. Planes de negocios superficiales, mal soportados técnica y financieramente. Muchos nuevos emprendimientos empresariales fracasan por que inician labores con alto endeudamiento, con significativos pasivos de corto plazo y niveles insuficientes de capital de trabajo, pero principalmente, por desconocer el sector en el cual se inserta la nueva empresa. En estas circunstancias es imposible evolucionar, porque, como es apenas natural, la maduración financiera, técnica y de mercado de todo negocio exige períodos prolongados antes de capitalizar adecuadas utilidades. Así sea el aprovechamiento de una cesantía derivada del retiro de un funcionario o de los ahorros de toda la vida de un grupo familiar, ya sea que se piense en la compra de un vehículo de transporte público como herramienta de inversión o la instalación de una tienda de barrio, o cualquier otro proyecto de alguna complejidad, se precisa en todo caso realizar estudios de preinversión, así sean superficiales, que permita al novel inversionista dimensionar adecuadamente el mercado, las inversiones necesarias, los costos de operación y, desde luego, todas las labores administrativas que le garanticen su adecuado funcionamiento. La estructuración de un proyecto permitirá simular y dimensionar tempranamente algunos acontecimientos que se presentarán en el futuro y facilitará la familiarización y aproximación con el entorno del negocio. El emprendedor tendrá que resolver cual es el tamaño adecuado para su empresa, el que se ajuste a su tecnología, a su estrategia, a sus finanzas y a sus mercados. Los conocimientos sobre el sector económico y del ámbito geográfico serán básicos para robustecer los argumentos a favor o en contra de la decisión de crear una nueva empresa. A algunos les parecerá un ejercicio inocuo que retarda la toma de una decisión, lo más importante dicen es montar el negocio lo más pronto posible. Un ejemplo muy frecuente en nuestro paisaje urbano se observa al visitar centros comerciales, donde el destino de los locales cambia en forma permanente. Estos cambios repentinos en el uso de los locales denuncian en alguna medida las decisiones tomadas sin conocer la naturaleza del negocio, en donde la improvisación prima sobre la planificación, con todas las consecuencias negativas sobre las finanzas del inversionista, sobre su estado de ánimo y su moral al sentirse frustrado por el fracaso. Triste observar el profundo desencanto y desesperanza de aquellos que ilusionados con una idea vaga tomaron la decisión de montar un negocio y que con nostalgia afirman ahora que se precipitaron, que no pensaron suficientemente, que hicieron cuantas alegres, que magnificaron los ingresos y subvaloraron los costos, que no conocían los rigores de la organización, que cedieron a las lisonjas de los proveedores, que relajaron su control y vigilancia sobre los créditos recibidos y concedidos, etc. 
La ignorancia y la mala preparación abren el camino del fracaso. La permanencia y crecimiento de una nueva empresa exige que el emprendedor, más allá de la buena voluntad, posea una serie de actitudes, habilidades, conocimientos y técnicas. Estas las puede adquirir previamente, en la medida que "estructura su proyecto", 0 aprenderlas en forma traumática durante la operación, con un costo tan grande que puede amenazar la supervivencia de la nueva empresa y el patrimonio de su propietario. La estructuración del proyecto no garantiza el éxito de la decisión, simplemente disminuyen el riesgo de fracaso.

La estructuración del proyecto (simulador de futuro) se constituye en el soporte adecuado para la elaboración de los "planes de negocios", herramienta indispensable para desarrollar los contactos y las negociaciones que apuntan hacia el nacimiento de nuevas empresas o la consolidación y ampliación de las existentes.

Se trata de nuevas unidades empresariales o nuevas líneas en las existentes, que surgen no de una espontánea manifestación de interés derivado de una observación repentina y superficial, sino aquellas que son el producto de ponderados estudios previos en donde se han dimensionado los riesgos y se han tomado medidas reales para mitigarlos y controlarlos. En este documento en ningún momento afirmaremos que el proceso de crear una empresa o una nueva línea en una ya existente, es una tarea fácil, no ofrecemos falsas expectativas, antes por el contrario, parece que toda la legislación se haya diseñado para estorbar y desestimular al emprendedor, la transferencia tecnológica es costosa y difícil de asimilar, los inversionistas no entregan sus recursos sin la seguridad que éstos sean cuidados y multiplicados adecuada y ventajosamente, las autoridades exigen el cumplimiento de todos los requisitos (en la mayoría de los casos inocuos y exagerados), los administradores tributarias celosos y necesitados de recaudos se tornan más eficientes. No queremos ofrecer "un camino fácil" para crear una empresa, tampoco ofertas de "lecciones rápidas" ni mucho menos, indudablemente estamos frente a un proceso complejo, donde el conocimiento, la persistencia y el esfuerzo son los ingredientes necesarios. Detrás de cualquier empresa exitosa independiente de su tamaño, existe un emprendedor esforzado y conocedor que gracias a su tenacidad y terquedad, paciencia y valor para enfrentar los muchos problemas no previstos ha logrado sobrevivir en un ambiente poco propicio. Es oportuno reiterar, que la maduración financiera, de mercado y organizacional se logra después de un período más o menos prolongado. La visión del nuevo emprendedor debe ser de mediano y largo plazo, en el inmediato y corto plazo es imposible consolidar un negocio. Una empresa corresponde a un compromiso de vida. Nuestra intención está orientada necesariamente de empresas formales que tienen que cumplir con los requisitos de ley (seguridad social, obligaciones tributarias, permisos y aprobaciones, capacidad de respaldar créditos, etc) y de organización, proceso éste que suele ser pausado y costoso, pero que es preciso asumir con toda la responsabilidad necesaria, so pena de encontrar más adelante obstáculos insalvables y frustrar prematuramente el desarrollo

\footnotetext{
${ }^{31}$ Información tomada de las Cámaras de Comercio en América Latina indican que de cada 100 emprendedores que deciden crear una empresa, tan sólo 15 logran ponerla en marcha y solamente 3 alcanzan operación más allá del tercer año.

32 La ignorancia es la causa principal de los fracasos empresariales. Nada resulta tan caro como la improvisación.

${ }^{33}$ La informalidad es espontánea, repentista e improvisada y surge de la necesidad de atender una situación perentoria y urgente, es precisamente lo contrario a las pautas que daremos en el presente documento.
} 
De ahí surge la necesidad de formar a las nuevas generaciones en un nuevo esquema, ya no el de futuro empleado sino de nuevo empresario. El "espíritu empresarial" que es el rótulo que se le da a esta nueva forma de concebir el futuro no se reproduce en forma espontánea, es preciso construirlo desde las edades más tempranas en el ambiente familiar y los pupitres del colegio.

Las universidades deben jugar un papel fundamental en éste nuevo propósito pues son los responsables de formar un nuevo profesional bien informado técnicamente y respetuoso de las reglas de juego acordadas por la sociedad (formación ética), pero también con la capacidad de crear a través del ejercicio de su profesión una unidad empresarial sólida capaz de dar albergue permanente a nuevos empleos en cumplimiento de su objetivo personal y social. Bien importante que las universidades aprovechen el gran caudal de ideas plasmadas en los trabajos de grado en algunos casos mal llamados "tesis" y faciliten a sus autores la posibilidad de llevarlas a cabo. Se precisa concentrar esfuerzos y buscar apoyo para que las ideas más prometedoras sean compensadas con recursos que permitan profundizar los estudios y llevarlas a niveles de prefactibilidad o factibilidad, y respaldados por éstos, elaborar los correspondientes "planes de negocio" que facilite las primeras pesquisas ante potenciales inversionistas 0 agentes de interés. Indudablemente, se precisa crear al interior de las diferentes unidades académicas o facultades de las universidades el espíritu empresarial, donde tanto docentes como directivos lo mismo que estudiantes observen en su universidad el mejor laboratorio para crear y dar los primeros pasos para desarrollar una empresa.

La universidad del futuro (en todas sus facultades), tendrá la tarea adicional de oficiar como "incubadora de empresas", por lo tanto tendrá que incorporar en sus planes de estudio nuevas asignaturas que faciliten el proceso, pero ante todo, permitir, impulsar, fomentar y descubrir ideas prometedoras que se puedan convertir en empresas exitosas.

Por otro lado, se espera que la nueva universidad sea el lugar desde donde se crean los conocimientos para que fluyan hacia donde están los estudiantes, complementando poco a poco el costoso formato presencial con el de universidad abierta, a distancia 0 universidad virtual.

Las estrategias para promover el desarrollo empresarial, como las incubadoras de empresas, los fondos de capital de riesgo, el crédito, los aportes de capital semilla, y otros mecanismos útiles deben estar acompañados necesariamente de una formación y capacitación de calidad orientada al talento emprendedor y creativo, a su espíritu de trabajo y conducta ética que les permita a través de la estructuración de proyectos concretar sus sueños y aspiraciones, y materializarlos en empresas exitosas 


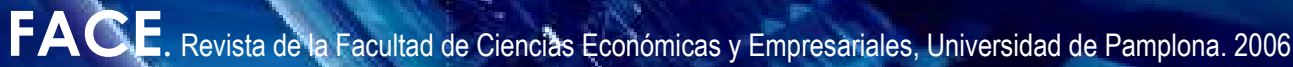

\section{Manifiesto de la fundación del Espíritu Emprendedor ${ }^{33}$}

Estamos convencidos de que en la sociedad existen muchas personas, hombre y mujeres, con habilidad para transformar sus sueños, sus problemas o sus oportunidades, en empresas viables. Cuando dichas personas dirigen empresas propias se llaman emprendedores (entrepreneur); pero también hay personas que trabajan para empresas ajenas, y lo hacen con espíritu emprendedor (intrapreneur):

Esas personas emprendedoras muchas veces ignoran sus habilidades y cuando las descubren, dudan en desarrollarlas plenamente, sea por temor, o sea por una conciencia equivocada respecto al apoyo de su comunidad hacia sus iniciativas.

Estamos convencidos de que una persona con espíritu emprendedor, llegará a su pleno desarrollo personal sólo si decide poner en acción sus potencialidades, dando libre curso a su talento emprendedor.

A esto declaran respectivamente los señores Robert Arcan y Bernard Lemaire: "Mi vida empezó a los treinta años, cuando inicié mi empresa"; sólo me siento a gusto cuando emprendo algo".

Estamos convencidos de que la creatividad de los hombres y de las mujeres es ilimitada, al igual que son ilimitadas las necesidades humanas.

La escasez de empresas duraderas se origina en la insuficiencia de emprendedores independientes y de empleados con espíritu emprendedor.

Tenemos la firme convicción de que las personas con cualidades emprendedoras, tienen generalmente éxito cuando se proponen:

1. Adquirir los conocimientos esenciales de administración;

2. Adquirir experiencia en el campo específico de la actividad que han elegido;

3. Utilizar los recursos disponibles;

4. Trabajar a su propio ritmo;

5. Disponer de suficiente capital inicial para arrancar un negocio y manejarlo por determinado tiempo. 
Afirmamos que las características distintivas de los emprendedores y de las personas con espíritu emprendedor son las siguientes:

1. Consideración de todas las facetas de un problema.

2. Necesidad imperiosa de realizar sus proyectos.

3. Interés innato más por las oportunidades que están a la mano, que por el análisis de los problemas.

Preferencia por dedicarse a proyectos en vez de buscar la notoriedad.

Desprecio por los fracasos y por las dificultades; éstos son enfrentados y controlados.

En opinión del señor Bernard Lamarre, las personas con espíritu emprendedor y los emprendedores, se identifican entre sí por su capacidad de análisis y cuestionamiento del orden establecido, y por su aprecio por el trabajo innovador.

Aseguramos que en una democracia, es la sociedad la que escoge a sus gobernantes, que nunca faltarán; en cuanto a los empresarios y a los emprendedores, la sociedad tiene sólo aquellos que se merece, ya que surgen por sí mismos (¿será por eso que frecuentemente son insuficientes?) Las acciones siguientes son indispensables para que una sociedad pueda disponer de empresarios y de gente con espíritu emprendedor:

1. Interpelar constantemente a hombres y mujeres, con el fin de descubrir empresarios y emprendedores;

2. Apoyar en forma positiva a la gente, sin marginar a nadie. Nunca rechazar, sin analizarlos, los proyectos innovadores, aunque a veces se salgan de lo convencional;

3. Apoyar a los empresarios y a los emprendedores que surjan, proporcionándoles: capacitación, acceso al capital inicial, apoyo publicitario, apoyo comunitario y demás apoyos. Finalmente, valorar como se lo merecen a aquellas personas que por sus iniciativas han transformado el entorno, mejorándolo continuamente.

Los empresarios y los emprendedores son valiosos para la sociedad, elemento que debe reconocerse y darle mérito. Sabemos perfectamente que no todos pueden ser empresarios o personas emprendedoras dentro de su fuente de trabajo. Pero debemos apoyar el espíritu emprendedor en nuestro medio.

Independientemente de nuestra función de padres, patronos, educadores, empleados, consumidores, contribuyentes, inversionistas o simples ciudadanos, siempre nos será posible demostrar apoyo y respaldo e inclusive nuestro reconocimiento a aquellas personas que por iniciativa propia crean empleos y riqueza. Los medios de comunicación social pueden desempeñar una función sumamente importante para el avance y desarrollo del espíritu emprendedor, especialmente de las empresas, proponiendo una cultura organizacional que apoye a la innovación. En la opinión del señor Claude Beland, del movimiento Desjardins, las actividades económicas deben apoyar los esfuerzos del hombre hacia lo positivo y descartar la lucha por la supremacía. 


\section{FACE}

Consideramos que en todos los grupos hay un potencial emprendedor latente. Si este potencial es convenientemente estimulado, es capaz de generar las empresas necesarias para una utilización correcta de los recursos humanos del medio. Así se crearán los empleos y la riqueza que garantizarán el desarrollo del grupo.

Esta convicción es la plataforma para que se den el inicio, el crecimiento y el perfeccionamiento del desarrollo. Estamos firmemente convencidos de que el espíritu emprendedor es y sigue siendo el motor que gradualmente pero con profundidad transformará a la sociedad. El espíritu emprendedor es, también, un excelente instrumento para integrar elementos humanos distanciados por raza, edad, sexo y grupos sociales.

Es nuestra convicción que el desarrollo local y de cada región se sustenta en el desarrollo de la pequeña y mediana empresa y en el espíritu emprendedor. Una sociedad emprendedora está profundamente centrada en el hombre.

Es misión del Estado contribuir a la creación de condiciones favorables y propicias a la eclosión y crecimiento de los emprendedores y de la gente con espíritu emprendedor, indispensables para el fortalecimiento de una región. Afirmamos que en regiones con pocos recursos materiales y con dificultades de acceso a los mercados, deben capitalizarse los recursos humanos: creatividad, imaginación, habilidades técnicas y artísticas.

En dichas regiones deben atacarse firmemente los problemas derivados de sus condiciones negativas. Hay que investigar para encontrar canales adecuados de distribución y apoyar a las empresas que tienen dificultades.

Son indispensables los programas de apoyo a las iniciativas emprendedoras orientadas al desarrollo regional.

Estamos convencidos de que el emprendedor con éxito es el mejor candidato para nuevos proyectos: conoce su mercado, tiene acceso al financiamiento y tiene credibilidad para seguir progresando.

Si este emprendedor carece de posibilidades o de intenciones para iniciar algo nuevo, siempre podrá desempeñarse como inversionista-tutor de otro más joven; y esto con dos opciones: en su propia empresa o en la de otro. Para un joven sin experiencia y sin recursos, el inversionista-tutor, puede incrementar significativamente sus posibilidades de éxito.

Consideramos que las grandes empresas son importantes, tanto para el desarrollo regional como para el desarrollo del espíritu emprendedor. La microempresa requiere empleados con espíritu emprendedor para capitalizar su riqueza mental, así como su creatividad científica y técnica.

La gran empresa debe mostrar interés para localizar y apoyar gente con ideas emprendedoras, cuyos proyectos sean compatibles con los de ella. 
En este sentido, opina el señor Bernard Lamarre, "los empleados emprendedores constituyen un factor determinante para el potencial competitivo de nuestras empresas". En opinión del señor Claude Boivin, ser emprendedor el algo difícil pero es también algo absolutamente vital. Por encima del interés propio de una empresa, está el interés general de la colectividad. Esta consideración debe impulsar a las empresas a comprender a sus empleados emprendedores que la abandonen para iniciar proyectos personales. En esta forma la microempresa valoriza, apoya, recibe y reta al emprendedor latente que se manifiesta de ella.

Consideramos que los empleados con espíritu emprendedor pueden llegar a ser excelentes empleados sólo si sus patrones les dan oportunidad de desarrollar sus posibilidades. El apoyarlos para independizarse debe ser un motivo de orgullo para el empresario con espíritu emprendedor. El desarrollo de la región debe ser valorizado por los patrones que apoyan esta iniciativa de sus empleados al empresariado. Esto les da imagen ante la colectividad, y en ocasiones, permite el ascenso de jóvenes que desean adquirir experiencia.

Consideramos que nuestra sociedad, en esta época de cambios acelerados, debe basarse en su potencial emprendedor para garantizar su progreso y su sobre vivencia. Nuestra región debe poder dominar convenientemente el proceso de gestación e implantación de nuevas empresas, logrando promover el acceso al capital y la disponibilidad de éste con el fin de facilitar las inversiones de empresas variadas y numerosas. Pues, no hay que olvidar que son las pequeñas empresas y las empresas en desarrollo las que crean empleos.

Estamos convencidos de que el emprendedor que desea permanecer en los negocios y crecer, debe desarrollar en forma prioritaria la calidad total de su empresa. Citamos a la señora Jeannine G. Word de la Compañía Guillevin Internacional: "Para el emprendedor moderno, deseosos de participar en el mercado ofreciendo calidad total, el cliente debe ser objeto de atención prioritaria". Para la señora Ginette Delisle: "La calidad total se vuelve una filosofía administrativa, una manera de pensar y de actuar, un proceso continuo de organización respecto al cliente desde el levantamiento del pedido hasta la entrega del producto".

En la búsqueda de la calidad total, el desarrollo tecnológico es importantísimo. Hay que recordarlo: la calidad debe permear todas las actividades humanas.

Desarrollar la calidad en nuestras empresas y en nuestra sociedad requiere calidad humana, capacidad de inventiva. Para la señora Blanchet: "Para que el desarrollo tecnológico crezca eficientemente, debe tener raices profundas en el recurso humano de la organización y estar integrado a la cultura organizacional. Para obtener dichos resultados el emprendedor debe apoyarse en la información y en la información de su personal, con excelente comunicación tanto interna como externa". En estas condiciones el desarrollo tecnológico se traduce en innovaciones y en el mejoramiento de la calidad total de la empresa. 


\section{FACE}
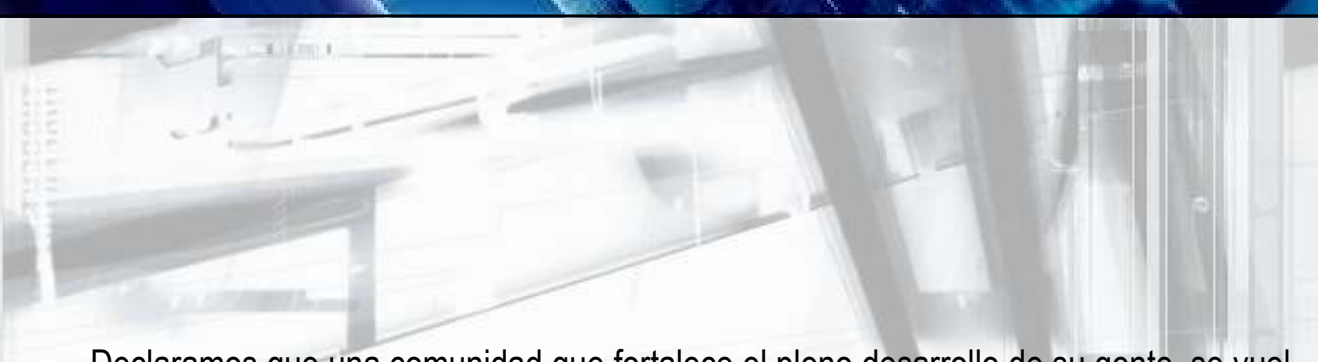

Declaramos que una comunidad que fortalece el pleno desarrollo de su gente, se vuelve rápidamente una comunidad emprendedora. Esta comunidad tendrá capacidad de lograr crear condiciones adecuadas para la utilización, a toda su capacidad, de los recursos humanos.

En esa forma se logrará el desarrollo gradual de una verdadera cultura empresarial.

Nosotros tenemos fe en que la educación sigue siendo el principal crisol en donde se moldean las generaciones sobre las que se sustentan la sociedad. Las escuelas transmiten primero los conocimientos y luego las actitudes y las conductas. Son también el vehículo de los valores y creencias con las que se construye una sociedad.

La educación primero se dirige al intelecto y luego al corazón y al ser humano en su totalidad. Para una comunidad humana, la cultura emprendedora sólo se hace posible cuando la educación escolarizada participa en este proyecto y se responsabiliza de él. Hay que merecer la sobrevivencia de un grupo o de un pueblo: tenemos que demostrar que nadie ha salido sobrando en el desarrollo demográfico.

Para lograr este objetivo, hay que desarrollar convenientemente el espíritu emprendedor con el fign de que se puedan ofrecer empleos adecuados a todos.

Tenemos conciencia de que nuestra sociedad constituye en occidente un ejemplo fuera de serie. En relación con el desarrollo del espíritu emprendedor, pasó, en menos de una generación, de una sociedad deficitaria a una sociedad autosuficiente. Inclusive es ahora capaz de transferir esta habilidad operativa a una buena parte del planeta.

Por último, estamos firmemente convencidos de que la misión que la Fundación del Espíritu Emprendedor se ha propuesto, en cuanto a identificar y acrecentar el potencial emprendedor de la gente, y de crear condiciones altamente favorables a su plena realización, responde a los requerimientos de nuestra época.

Hacemos hincapié en que esta organización, que es provisional, sólo debe durar el tiempo necesario para despertar las posibilidades latentes y que no se propone ningún tipo de ejercicio del poder. Nuestra Fundación sólo quiere ser un factor complementario al servicio de los recursos existentes. 


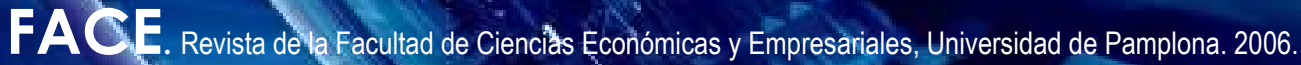

\section{$+2$

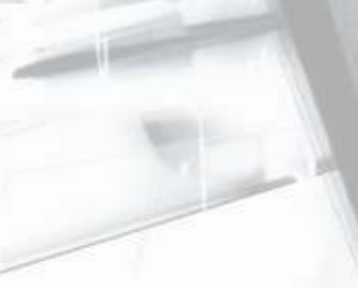 \\ Calidad, Productividad y Costos: Análisis de relaciones entre estos tres conceptos}

46

HAYDEÉ CECILIA RINCÓN DE PARRA

Facultad de Ciencias Económicas y Sociales Universidad de los Andes E-mail:haydocc@yahoo.com

\section{RESUMEN}

La economía mundial del presente, y particular la economía Venezolana es cada vez mas compleja, difícil, interdependiente y competitiva. Dentro de este escenario donde las dificultades tienden a ser condiciones normales de trabajo, la mejor respuesta a las condiciones económicas y de producción es la calidad, la productividad y la disminución de los costos. Con el presente trabajo se tiene como propósito contribuir a aclarar el sentido de dirección de la empresa en materia de calidad, productividad y costos tomando como referencia las relaciones que existen entre estos tres conceptos. Se refiere que una de las relaciones viene dada por: a mayor calidad, mayor productividad y menores costos.
Palabras Claves:

Competitividad.

Calidad.

Productividad.

Eficiencia.

Efectividad.

Costos. 


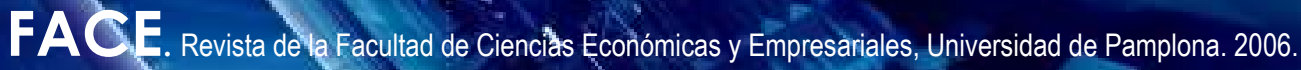

\section{7}

\section{Calidad, Productividad y Costos: Análisis de relaciones entre estos tres conceptos}

HAYDEÉ CECILIA RINCÓN DE PARRA

Facultad de Ciencias Económicas y Sociales Universidad de los Andes E-mail:haydocc@yahoo.com

\section{INTRODUCCIÓN}

Uno de los signos mas característicos de la sociedad de hoy es sin duda alguna la preocupación explicita la por calidad, productividad y los costos en todos los ámbitos de la economía internacional. Sin embargo, esta preocupación ha adquirido diferentes matices en función del sector de la economía en cuestión y del contexto nacional del que se trate.

En el caso de la economía Venezolana, esta se encuentra signada por las limitaciones y retos que la modelan y condicionan, tienen la oportunidad histórica de desarrollarse hacia niveles cuantitativos y cualitativamente superiores, mediante la gestión eficiente de sus propias fuerzas productivas y el esfuerzo de todos los sectores involucrados en la generación interna de trabajo y riqueza. Se considera que la gerencia actual debe sufrir cambios de enfoque, de expectativas y bases conceptuales con relación a la concepción global que se tiene de la empresa y, en particular, de su clima humano y cultural.

Con el presente trabajo se tiene como propósito contribuir a aclarar el sentido de dirección de la empresa en materia de calidad, productividad y costos tomando como referencia las relaciones que existen entre estos tres conceptos. Se refiere que una de las relaciones viene dada por: a mayor calidad, mayor productividad y menores costos. 


\section{FACE -}

\section{Calidad, Productividad y Costos}

Este trío de conceptos se puede analizar como un todo prácticamente inseparable, por cuanto el cambio de uno cualquiera de los elementos afecta los otros dos. Durante mucho tiempo se hizo énfasis en la mejora, de la productividad, bajo el supuesto de que, como consecuencia natural, se incrementarían los niveles de calidad. La evidencia demuestra ahora que el camino es exactamente el opuesto. Los incrementos de productividad no solo no conducen definitivamente hacia la mejora de calidad; sino que, incluso, la pueden deteriorar. Se propone entonces a la luz de la experiencia, que se de prioridad a las estrategias de garantía de calidad, las cuales, en consecuencia, incrementan la productividad y minimizan los costos, y no a las inversa.

Al respecto, Blanco (1999) señala que a veces las empresas tratan de mejorar la productividad del trabajo mediante el pago de incentivos económicos individuales por el cumplimiento de ciertas metas de producción. En muchos de estos casos, si los trabajadores no son consientes de la importancia de la calidad y la optimización de los costos, realiza las actividades de manera rápida y descuidan detalles importantes en los productos que pueden afectar la confiabilidad de estos, y, por ende, su competitividad. En otras ocasiones, las empresas fabrican productos de excelente calidad con la máxima eficiencia y ahorro de recursos, pero con precios que no son competitivos en el mercado internacional. Por esta razón de esa inseparabilidad de los tres aspectos objeto de estudio (calidad, productividad y costos), se considera que es el momento de establecer estrategias gerenciales. Una de estas estrategias estaría orientada al análisis de estos tres conceptos de manera integral.

Antes de iniciar el análisis específico de la relación de los tres elementos, conviene establecer con claridad algunos de los conceptos fundamentales y la interrelación entre los mismos, tal y como serán interpretados en el resto de este trabajo, a saber: competitividad, calidad, productividad, eficiencia y efectividad, y costos. 


\section{FACE. Revista de la Facultad de Cienciass Económicas y Empresariales, Universidad de Pamplona. 2006.}

\section{La Competitividad}

Con esta palabra se quiere significar que los bienes y servicios que provienen de los sectores productivos, deben tener la oportunidad de competir exitosamente en el mercado internacional. Son productos competitivos, en categoría global, aquellos cuyos precios corresponden a la franja de mercado estimada, los que tienen calidad certifica$\mathrm{da}$, los que entregan oportunamente, los que incluyan innovaciones fruto del conocimiento y la tecnología y los que han sido hechos pensando en el cliente, usuario o consumidor, con la confianza y duración especificadas y garantizadas, además de un conveniente servicio de mantenimiento y postventa.

Los productos competitivos son los que cumplen especificaciones universales y se pueden vender en el mercado internacional sin ningún problema; es decir, los bienes y servicios deben satisfacer normas internacionales de competitividad.

Ahora bien, el reto de transformar productos tradicionalmente protegidos por barreras arancelarias y competitivas en el medio regional en productos comercializables en el mercado mundial, es muy grande ¿Cómo lograrlo?

De acuerdo con Blanco, lo primero que hay que hacer es un diagnostico del mercado y la competencia. Se debe, mediante un profundo conocimiento del funcionamiento del mercado internacional, conocer cuales son las necesidades y expectativas de los clientes en los diferentes países, qué compañías conforman la competencia y como se comportan, cuales son las principales tendencias del mercado, cuales son los segmentos del mercado con mejores oportunidades, como deben ser los precios, únicos o diferenciados; si existen restricciones arancelarias o mecanismos de subsidio para los productos de la competencia, etc.

Esta fase de diagnóstico del mercado y de la competencia, el uso de tecnologías modernas de comunicaciones e informática se hace indispensable. Actualmente existe un buen numero de empresas que venden la información comercial, completamente actualizada, de carácter internacional sobre el tipo de productos o servicios, precios, cantidad, sitios y empresas que los demandan. 


\section{FACE}

\section{La Competitividad}

La información se puede comprar a estas empresas o, si están en posibilidad, es mucho mejor crear las fuentes propias de datos. La oportunidad que brinda Internet actualmente, y que se incrementara en un futuro no muy lejano, permite este tipo de actividad. En el caso Venezolano, vale la pena mencionar la labor desarrollada por el Banco del Exterior y el Ministerio de Industria y Comercio para ayudar a los empresarios a efectuar sus exportaciones.

En segundo lugar, los sistemas lógicos necesarios para la competencia deben ser capaces de brindar información instantánea, entre otras, del estado de los perdidos, de su ubicación, de las cantidades, del sistema de empaque, del peso y de las fechas precisas de entrega. Se debe conocer la documentación necesaria de cada país, los aranceles, las restricciones y barreras de cada región. Las redes de transporte y los sistemas de carga por emplear, tanto interna como externamente, deben ser altamente eficientes para evitar deterioros, perdidas, etc, y para garantizar la entrega de las mercancías a tiempo, y en los sitios previamente establecidos. En tercer lugar los productos de manufactura deben producir en excelentes términos de calidad, productividad y oportunidad. Los sistemas de planeaciòn, programación y control de producción deben ser capaces de proporcionar información oportuna y veraz de las materias primas, los procesos, el estado de las ordenes de pedido, las compras, los costos, los precios, las fechas de entrega, los inventarios disponibles, etc. Es importante señalar que todo el personal debe estar comprometido con la competitividad como estrategia de supervivencia de la empresa; por lo tanto, los empresarios deben estar consientes de quienes son sus clientes, de cuales son sus necesidades a satisfacer y de los precios objetivos (precios planeados) que harán vendibles sus productos o servicios en el mercado. 


\section{FACE -}

\section{Calidad}

Definir en que consiste la calidad de un producto o servicio es algo complicado. Una primera idea del término calidad es: siempre hay una mejor forma de hacer las cosas. Una frase corta y sencilla, pero aplicable hasta en los procesos más complejos que puedan existir en el mundo entero.

Así como esta, existen infinidad de frases que podrían explicar muy brevemente en que consiste el mejoramiento continuo de los procesos de trabajo; que es lo que en realidad busca la calidad de gestión mediante una definición, cuantificación, eliminación y prevención del desperdicio; par lograr un aumento de la productividad y una calidad y excelencia en los productos. Pero lo importante no es conocer de una manera fría y técnica lo que encierra esta definición, si no ir un poco más allá, internalizar dentro de cada uno, lo que significa. Un cambio de actitud tomando conciencia de que la idea no es formular utopías, sino hacerlas realidad siendo flexibles en nuestra mentalidad; solo así se tendrá la seguridad de ser capaz de hacerlo hoy mejor que ayer y mañana mejor que hoy.

Desde el punto de vista de adaptación, las empresas enfrentan hoy un constante cambio en económico. En consecuencia, deberían estar mejor preparadas operativas y gerencialmente para afrontar tales cambios. Por lo general en la mayoría de las empresas (caso Venezolano) se aplica el control retro-alimentativo; es decir, se espera tener el producto terminado para saber si se cumple con ellos, hay que corregirlo o simplemente se pierde y hay que intentarlo de nuevo. Tal vez si se obtiene un aumento de calidad; pero, al mismo tiempo, se aumenta el desperdicio y los costos, se disminuye la productividad, y las posibilidades de competir en los mercados regionales o internacionales son muy remotas.

"El termino calidad, por lo general lo asociamos con productos o servicios excelentes, que satisfacen nuestra expectativas $y$, mas aun, las rebasan. Tales expectativas se definen en función del uso que se dará al producto o servicio en cuestión y de su respectivo precio de venta" (Besterfield, 1995, pag 1). 


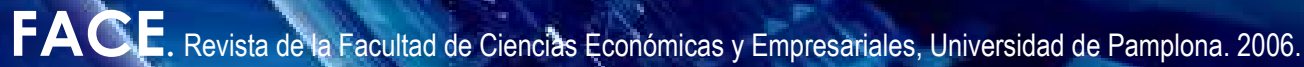

\section{Calidad}

"Con bastante frecuencia, calidad quiere decir conformidad con los planos y especificaciones y el control de calidad determina si el producto es conforme (...) Calidad se utiliza en un sentido mas amplio y significa adecuación para el uso por parte del usuario (...) y abarca tanto la calidad de diseño, como la calidad de conformidad" (Campanella, 1992, Pág. 163).

"Un producto de calidad debe cumplir sus especificaciones metrológicas, debe ser adecuado para el uso que se le ha de dar, debe ser confiable u durable, no debe presentar falla o deficiencias, debe tener un apropiado servicio de mantenimiento, repuestos y posventa, se debe entregar a tiempo, en las cantidades precisas ordenadas, etc." (Campanella, 1992, Pág. 163).

"Un producto de calidad debe cumplir las especificaciones metrológicas, debe ser adecuado para el uso que se le ha de dar, debe ser confiable y durable, no debe presentar fallas o deficiencias, debe tener un apropiado servicio de mantenimiento, repuestos y postventa, se debe entregar a tiempo, en las cantidades precisas ordenadas, etc." (Blanco, 1999, Pág. 18).

Juran (1990) enseña que la calidad de un producto no es el resultado del azar o de la buena de la buena suerte, sino el fruto del cumplimiento de todo un proceso de planificación. Conocer cuales son la necesidades y expectativas del cliente es quizá la parte mas difícil del ciclo de calidad. Sin embargo, actualmente existen herramientas metodológicas que ayudan a traducir las necesidades del cliente a especificaciones de diseño y producción. Si se planifica la calida y se produce de acuerdo con los planes, los productos, al final, resultaran de buena calidad. 


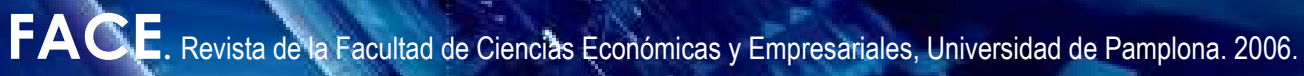

\section{Calidad}

Se debe tener en cuenta que las características de la calidad se identifican, en primer lugar a través de la investigación de mercados que muestran lo que quieren los consumidores (el enfoque de la calidad basado en el usuario final). Estas características se trasladan a los atributos que debe tener el producto (el enfoque basado en el producto). Después, el proceso de fabricación asegura que los productos se realizan cumpliendo de forma precisa las especificaciones (enfoque basa do en la producción).

En resumen, un proceso que omita alguno de estos puntos no dará como resultado un producto que satisfaga al cliente. Es de hacer notar que la calidad comienza con la gente que elabora en la empresa. Para cualquier gerente el concepto y las herramientas de la calidad total deben ser principios nobles para poder convencer al más escéptico. No puede existir un gerente que no desee que su empresa entre en un proceso continuo de mejora, para poder satisfacer a plenitud las necesidades de sus clientes. Todas las personas en la empresa deben comprender que la calidad no es una moda, sino una estrategia de supervivencia, que la empresa no puede ser competitiva en tanto no satisfaga al cliente, mientras no elimine el desperdicio, mientras no trabaje con parámetros de productividad y mientras no optimice los costos. Pero, ¿Qué se entiende por calidad total? ¿Por qué se hace énfasis sobre la calidad? ¿Qué se pretende con el mejoramiento de la calidad?.

"La calidad total se entiende como una estrategia competitiva que la alta gerencia decidió implantar para satisfacer mejor las necesidades de los clientes y aumentar el retorno de la inversión" (Alexander, 1994, Pág. 1).

Homgren, et al (1996 Pág. 794) señalan que "se hace énfasis en la calidad, por uqe los costos asociados con la calidad son considerables. Los costos de calidad varian de $15 \%$ a $20 \%$ de los ingresos de ventas de muchas organizaciones, y con un buen programa de calidad, se pueden obtener ahorros sustanciales e ingresos mas elevados". 


\section{FACE. Revista de la Facultad de Cienciass Económicas y Empresariales, Universidad de Pamplona. 2006.}

\section{Calidad}

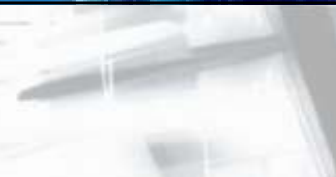

Para Heizer y Render (1997) la calidad es muy importante e el ambito de la empresa por que la afecta en cuatro aspectos fundamentales:

1. Costos y cuota de mercado: la mejora de calidad lleva a la vez a mejorar la cuota de mercado y a rebajar los costos.

2. Reputación de la empresa: la calidad aparecerá en la percepción de los nuevos productos, en las practicas de empleo y en la relación con lo proveedores.

3. Seguridad de los productos: en el sentido de las organizaciones no deben diseñar, producir y distribuir producto o servicios, capaces de producir daños materiales o personales durante su uso (el uso de los cauchos defectuosos fabricados por la Firestone y utilizados por la Ford en uno de sus productos).

4. Implicaciones internacionales: para poder competir de una manera eficaz en una economía mundial, los producto deben ajustarse a unas exigencias globales de calidad y precios.

Finalmente, se tiene que la calidad total, como ha sido comprobada por la reacción en Cadena Deming (Deming, 1989) lleva a una organización, por el medio de mejoramiento continuo, a aumentar su productividad, incrementar su mercado de clientes y a reducir costos.

\section{Productividad}

En cualquier discusión económica, la productividad ocupa un primer plano en las apreciaciones tanto de los dirigentes políticos como de los hombres de negocios. El concepto de productividad merece pues, una consideración seria y detenida. Precisa, para lograrla, examinar uno tras otro los criterios de quienes están interesados en el desarrollo de esta herramienta gerencial.

Desde el punto de vista económico, el elemento final en el cual el inversionista se forma su juicio sobre el progreso de su empresa es, tradicionalmente, el último renglón del estado de ganancias y pérdidas: la utilidad. Lograr una tendencia favorable de esta con relación a un rendimiento satisfactorio del capital invertido es la meta fina. 


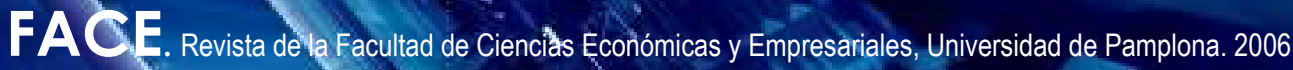

\section{Productividad}

La utilidad refleja el resultado neto de los esfuerzos de la gerencia en planear y desarrollar la producción, las compras, el mercadeo, los recursos humanos, las transacciones financieras y los otros factores de las operaciones de la empresa.

Sin embargo, a largo plazo, el factor mas importante para el buen desenvolvimiento de una empresa es su adaptación al progreso tecnológico y a los avances de la administración científica, lo que resulta en nuevos y mejores productos, de mayor calidad, a precios mas accesibles al consumidor. El progreso de una empresa en este sentido, se refleja en algo que se puede llamar reducción de los costos reales de producción; es decir, asumir la cantidad física de los insumos en forma de materia prima, trabajo y capital, por unidad de producto; en otras palabras en utilizar mejor los recursos empleados en la producción, y esto se traduce en un aumento de la productividad.

\section{¿Qué se entiende por productividad y como se mide?}

El desarrollo del pensamiento económico y gerencial sobre la productividad conduce a entenderla como un fenómeno complejo, que reviste una importancia estratégica de primer orden para las empresas que aspiran convertirse en sobresalientes, y, así permanecer en el tiempo.

El concepto de productividad, si bien muy popular en los últimos años, todavía esta envuelto en un manto de confusión. Para algunos autores, la productividad se concentra en la medición de indicadores, cuya variación en el tiempo evidencia el comportamiento de empresa y el estimulo en el proceso de toma de decisiones.

Para otros, la medición es menos importante y recomiendan dedicar los esfuerzos en la motivación y participación de los trabajadores. Consideran que si se logra que cada persona actúe más productivamente, el resultado global se traduce necesariamente en una empresa mas productiva. 


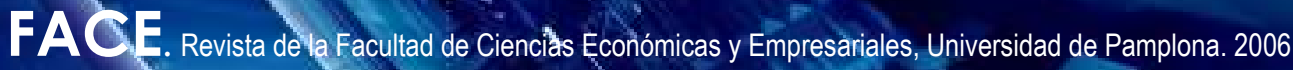

\section{Productividad}

Existen muchas formas de medir y analizar la productividad de la empresa; depende de los objetivos que se busquen. Según Prokopenko (1989), citado por Blanco (1999), algunos métodos sencillos y prácticos de analizar la productividad en la empresa son los siguientes: a) Medida de la productividad de los trabajadores. b) Sistemas de medición para planificar y analizar las necesidades de mano de obra en las unidades de producción. c) Sistemas de medición de la productividad del trabajo orientados a la estructura del uso de los recursos de mano de obra. $Y$ d) Productividad del valor agregado de la empresa.

Sobre el particular, Blanco argumenta que tradicionalmente la productividad total de la empresa, se ha visualizado como razón matemática entre el valor de todos los productos y servicios fabricados o prestados y el valor de todos los recursos utilizados en hacer el producto o prestar el servicio en un intervalo de tiempo dado, si esta razón resulta mayor que la unidad, indica que de alguna manera se esta agregando valor a los recursos durante la producción en otras palabras, que la salida del sistema productivo es mayo r que su entrada.

La mayor dificultad para esta medición la constituye la diversidad de unidades de medidas que se utilizan, además de los instrumentos y tiempo que son necesarios para hacerla, de ahí la necesidad de trabajar con unidades comunes tanto para los productos como para los recursos.

Las más utilizadas son las unidades monetarias y las horas-planta. Entre las dos, para efectos prácticos, la de mayor utilidad es la unidad monetaria. Por lo tanto, la información de costos de los recursos utilizados en la producción y precios de venta actualizados de los productos debe estar disponible.

Sin embargo, hay que tener cuidado de deflactar las cifras con índices como el de precios al de consumidor, el de inflación etc., (generalmente suministrados por entidades oficiales) para evitar confusiones con la rentabilidad. 


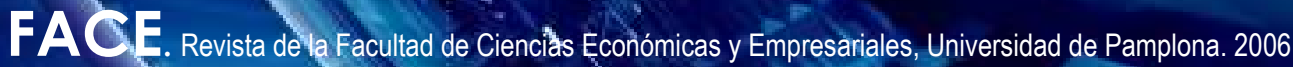

\section{Eficiencia y Efectividad}

La eficiencia es un concepto que con frecuencia se utiliza como sinónimo de productividad; se resumir como la utilización optima de los recursos. Un trabajador eficiente debe utilizar los materiales con el mínimo de: Según Blanco, prácticamente cada producto, cada recurso, tiene su propia unidad de medida.

El producto puede estar medido en número de unidades terminadas y utilizar un porcentaje de terminación para medir los productos en proceso. Los recursos humanos se pueden medir en horas dedicadas a la elaboración de productos. Los materiales se miden en unidades de cantidad, volumen, peso, etc. (láminas, toneladas, metros cúbicos, cuadrados o lineales, etc.) según las características especificas de estos, los servicios públicos se pueden medir en metros cúbicos, en el caso del acueducto, kilovatios en el caso de la energía, numero de llamadas, tiempo y destino en el caso de los teléfonos, etc. el capital se mide en unidades monetarias como el bolívar, el dólar, el peso, etc.

Desperdicio; emplear el mínimo tiempo posible en la producción, sin deteriorar la calidad del producto; utilizar los servicios (electricidad, agua, gas, etc.) en las cantidades necesarias, sin desperdicio, y utilizar los medios tecnológicos (maquinas, equipos, herramientas, etc.) de manera tal que no se deterioren mas de lo normal. El uso y conocimiento por pare del operario de los estándares de producción le permitirán saber que sucede y como aprovechar de manera optima sus recursos. El operario debe participar en la elaboración de los estándares y en la medición de su propia eficiencia, pero esto solo se puede lograr cuando existe participación, compromiso, logro y reconocimiento. 


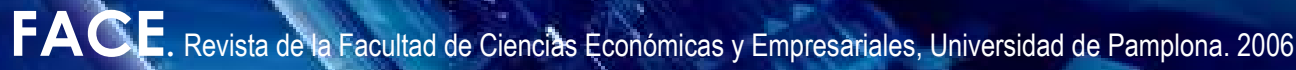

\section{Eficiencia y Efectividad}

La efectividad se define como el logro exitoso de objetivos establecidos. Es el grado en que se satisfacen las necesidades del cliente. De alguna manera el trabajador debe estar enterado de la forma como su contribución al valor agregado del producto sirve para satisfacer las necesidades y exigencias de los clientes.

El trabajador debe conocer que ha contribuido a que el producto final sea de la calidad esperada por el cliente, y este conocimiento lo hace sentir mejor. Es por eso, que la capacitación debe ser permanente para tratar de cumplir dos objetivos: la calidad del producto o servicio y la satisfacción del trabajador al tomar conciencia de que esta haciendo las cosas bien, que contribuye de esta manera a la supervivencia de la empresa y al mejoramiento de la economía del país.

Es importante destacar estos conceptos en tanto que la productividad es una medida de desempeño por que esta orientada hacia el cliente (efectividad) y, porque mide los aspectos importantes de la producción (eficiencia). Una de las expresiones de la efectividad es la calidad. De esta manera, la calidad se relaciona no solo con los costos y la productividad, sino que forma parte del concepto de efectividad. Es posible conseguir efectividad con una baja 0 alta productividad, 0 se puede ser altamente productivo $\sin$ ser efectivo.

\section{Costos}

Junto con la calidad y la productividad, los costos juegan un papel importante en la estrategia competitiva de la empresa. Pero, ¿Qué son los costos? A grosso modo, hablar de costos implica hablar de una serie de recursos necesarios para alcanzar una meta y/o lograr un objetivo. En este contexto, los costos se pueden generar por las actividades que hay que desarrollar en investigación y desarrollo, diseño e ingeniería, compras y almacenamiento, producción, (materiales, mano de obra y costos indirectos de fabricación), mercadeo (promoción, publicidad, ventas, distribución del producto, etc.), servicio al cliente, gestión de recursos humanos, administración, gestión de recursos financieros y otras. 


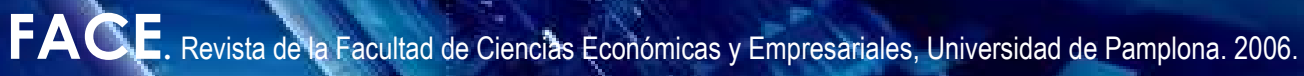

\section{Costos}

Los conceptos de costos y gastos, desde el punto de vista de las finanzas, significan lo mismo, ambos términos se manejan cuando se habla de desembolso de dinero. Sin embargo, desde el punto de vista contable, algunos consideran que son diferentes, en el sentido de que el concepto de costos lo asocian con desembolsos de dinero o no, aplicados a los procesos de manufactura (costos de producción) que se aplican a los ingresos del periodo de una manera mediata y paulatina. Los gastos lo asocian con desembolsos de dinero o no, que no se adicionan al valor de los productos elaborados, sino que se cargan a los ingresos del periodo de una manera directa, inmediata y en su totalidad.

En cualquiera de los casos, tanto los costos como los gatos influyen de una manera directa en los ingresos afectando la utilidad del periodo respectivo, por tanto, los costos de producción se deben sumar a los gastos (costos del periodo) y así determinar un costo total de operación.

Uno de los objetivos básicos de los sistemas de costos es la determinación de los costos unitarios de producción, para la fijación de los precios este aspecto debe ser analizado y estudiado en lo siguiente: tradicionalmente solo una vez terminado el bien o servicio, se podían determinar los costos de producción, los gastos de administración y ventas, los impuestos y la utilidad; y en función de ello se calculaba el precio de los productos 0 servicios, que podría no ser competitivo en el mercado.

Hoy día se esta hablando de un costo objetivo o costo meta (Target Cost) impulsado por los japoneses. Por medio del Target Cost se pasa de una gestión de la estabilidad a una gestión del cambio. Al respecto, Rincón (2000) señala que las empresas para lograra nuevos mercados o penetrar indeterminados nichos de mercados, deben garantizar la calidad total de los productos, en términos de, satisfacer las necesidades de los clientes, ofrecer un precio que asegure la demanda en el mercado y que les permita un beneficio adecuado, para finalmente, determinar el costo máximo en que pueden incurrir. 


\section{FACE}

\section{Costos}

¿Qué hacen los japoneses al respecto? De acuerdo con Moden (1992) citado por Blanco (1999), los japoneses determinan las necesidades del consumidor y, en función de ello, fabrican un producto que les permita hacer un nicho en el mercado, pronosticando los precios y el comportamiento de los costos. Parten de un precio meta competitivo en el mercado; pronostican una utilidad, estiman los impuestos, plantean metas para gastos de administración y ventas, y establecen metas de los costos de producción. En cualquier etapa del proceso toman la decisión de no producir o de buscar alternativas para reducir los costos con el fin de lograr los precios objetivos.

Con el enfoque antes descrito, las empresas pueden lograr mejoras en la calidad y en la productividad, disminución en los costos y, por consiguiente, ser más competitiva, es decir, mantener nichos de mercado y conquistar nuevos mercados.

\section{Relaciones entre Calidad, Productividad y Costos}

Aun cuando a lo largo de este trabajo de han esbozado algunas relaciones entre los conceptos estudiados, se exponen a continuación, las posibles relaciones que existen entre la calidad y los costos; entre la productividad y los costos; y entre la calidad y la productividad y, por ultimo, un resumen de las relaciones entre los tres: calidad, productividad y costos.

\section{Calidad y Costos}

El mejoramiento de la calidad y la reducción de costos son compatibles. La calidad aplicada a los procesos de trabajo induce a la reducción de costos. Una mejora de la calidad en los procesos de trabajo respecto a la fabricación, venta y distribución de productos 0 servicios, origina como resultado una menor cantidad de errores, de productos defectuosos y de repetición del trabajo, corta el tiempo total del ciclo y reduce o elimina el desperdicio de recursos disminuyendo por tanto el costo total de las operaciones. 


\section{FACE}

\section{Calidad y Costos}

Ullman (1985), citado por Ruelas (1993), señala la existencia de percepción no justificada de la calidad y el control de los costos son incompatibles debido a que, por una parte, la calidad cuesta y, por la otra, los controles de costos afectan la calidad. Este autor demuestra que la aparente incompatibilidad no existe como afirmación absoluta. Aunque existen algunos factores tendentes a mejorar la calidad, que sí impactan los costos, como por ejemplo la inversión en bienes de capital, aspectos relacionados con la mano de obra intensiva, que pueden mejorar la calidad de manera sustantiva sin afectar, necesariamente, de una manera negativa los costos.

En resumen, el mejoramiento de la calidad y la reducción de costos se deben llevar acabo simultáneamente, con el fin de satisfacer los requerimientos del cliente de hoy. Las empresas deben abandonar la filosofía de que una mejor calidad cuesta más dinero.

Lo expuesto, recoge lo planteado por Imai (1998, Pág. 46): "en un momento en que los clientes exigen un $\mathrm{QCD}^{2}$ mejor, la gerencia debe hacer énfasis en la prioridad adecuada para lograr estos tres elementos: ¡Primero la calidad! ¡Resístase a la tentación de reducir costos a expensas de la calidad! Y, ¡no sacrifique calidad por entrega! ”.

\section{Productividad y Costos}

Las relaciones entre productividad y costos, de acuerdo con el análisis conceptual sobre productividad expuesto en las sesiones anteriores, resultan obvias en las propias definiciones. La productividad mejora cuando una menor cantidad de insumos, que significa también menores costos, genera la misma producción. si se asume la existencia de una producción mayor con un numero

${ }^{2}$ QCD: siglas que en ingles se refieren a Quality, Cost, Delivery, que traducidas a lespañol significan: Calidad, Costos y Entrega. Este último término se refiere a la entrega puntual del volumen del producto o servicios, par satisfacer las necesidades del cliente $(\mathrm{N}$. del A.). 


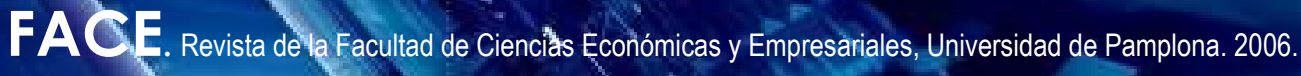

\section{Productividad y Costos}

menor de insumos, lo cual significa también menores costos y productividad, es analizar las dos posibles alternativas que se tienen cuando se considera una política de contención de costos. Una de estas alternativas, no necesariamente excluyente de la otra, es establecer restricciones para la utilización de os insumos con lo cual, evidentemente, los costos diminuyen. La otra es disminuirlos; pero no a expensas del establecimiento de restricciones, si no como resultado de un incremento de la productividad o eficiencia, pues de esta manera se obtienen más unidades de productos por el mismo costo. El efecto entonces es que, en términos relativos, el costo disminuye, y esto seria directamente inverso ante una indiscriminada utilización de insumos o una baja productividad 0 eficiencia, en cuyo caso los costos evidentemente se incrementan (Ruelas, 1993).

\section{Calidad y Productividad}

Para Deming (1989) la relación es muy clara, debido a que las estrategias para mejorar la claridad conducen hacia una minimización de los costos, debido a: la disminución en el numero de procedimientos que deben repetirse por haberse realizado mal la primara vez, la disminución en los retrasos de procesos y procedimientos, la mejor utilización de los recursos, etc. de aquí, que al mejorar calidad y evitar así las situaciones que se han mencionado, se tienden hacia un incruento de la productividad.

Existe una aparente relación reciproca entre calidad y productividad. Todo depende del énfasis en la estrategia que debe adoptarse para mejorar tanto la calidad como la productividad al mismo tiempo.

En apariencia, y durante mucho tiempo, el énfasis se puso casi de manera exclusiva en la productividad, como si al mejorar ésta, se incrementaría, en consecuencia, el nivel de calidad. El resultado no fue el esperado, debido a que se mejoro la productividad pero en algunos casos hubo un deterioro de la calidad. Si se da prioridad a la calidad sobre la productividad, el resultado es diferente, porque las estrategias orientadas hacia el 


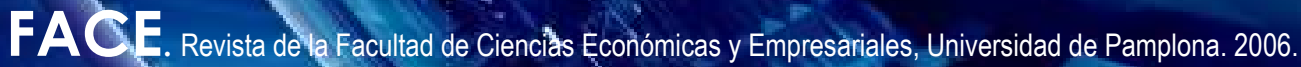

mejoramiento de la calidad, significan ahorros importantes en los procesos de producción, que conducen a una mejora de la productividad.

\section{Resumen de las relaciones Calidad, Productividad y Costos}

Las relaciones entre calidad, productividad y costos pueden resumirse en las siguientes posibilidades que parecerían ser las mas obvias:

A) A mayor calidad, mayor productividad y menores costos.

B) A mayor productividad, menores costos y menor calidad, si es que las estrategias para incrementar la productividad no consideran su impacto en la calidad.

C) A menor productividad, mayores cortos y, muy probablemente, menor calidad.

D) Si, a pesar de existir baja productividad, se intenta elevar la calidad, es muy probable que los costos se incrementen notoriamente para compensar la baja productividad. Aunque parezca redundante, es indispensable insistir en que los incrementos en la productividad conducirán, por definición, a la disminución de los costos, pero no siempre a los incrementos de la calidad. 


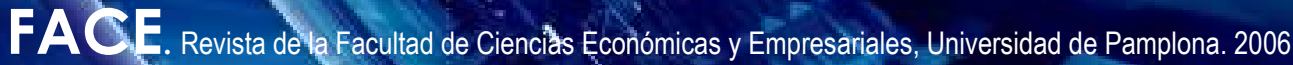

\section{Conclusiones}

La productividad es la medida de eficiencia y eficacia con que funciona el sistema de operaciones. Por ello, el sentido, la razón de ser y la importancia del concepto de productividad en la empresa, están en función de la continuidad y sobrevivencia en un ambiente de crisis y de competitividad. Las consideraciones anteriores, aunada a la oferta de productos y servicios que satisfagan (calidad), constituyen valores inherentes o rectotes que guían y orientan la esencia misma de la empresa, en el entendimiento de que tales valores son comprendidos y compartidos por cada uno de os actores dentro de la organización.

La productividad y la calidad son las armas de las que una organización dispone, a fin de no utilizar los incrementos de precios para minimizar los efectos negativos del aumento constante de los costos totales de operación. Se deben buscar, de una manera razonable y racional, incrementos en la productividad siempre cuando estos se consideren como parte de una estrategia global de garantía de calidad. A veces, la búsqueda de la productividad, a pesar de que puede disminuir los costos, deteriora la calidad.

En resumen, los conceptos de calidad, productividad y costos se deben manejar dentro de las organizaciones, de una manera integral (holística), que haga posible que el todo sea superior a la suma de las partes (sinergia). 


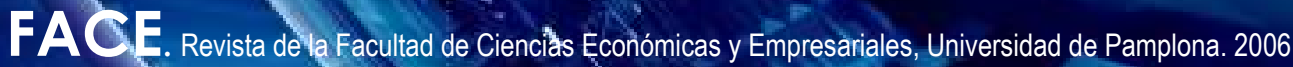

\section{Referencias Bibliográficas}

1. Alexander, Alberto G. (1994). "La Mala Calidad y su Costo". Por Addison-Wesley Iberoamericana. Wilmington, Delaware, U.S.A.

2. Besterfield, Dale H. (1995). "Control de Calidad". Editorial Prentice Hall Hipanoamericana, S.A., México.

3. Blanco, Luis E. (1999). "Productividad, Factor Estratégico de Competitividad a Nivel Global". Escuela Colombiana de Ingeniería, Bogota.

4. Campanella, Jack. (1992). "Principios de los Costos de la Calidad". Ediciones Diaz de Santos, S.A., Madrid.

5. Horngren, Charles; Foster George y Datar, Srikant. (1996). "Contabilidad de Costos". Un Enfoque de Gerencia”. Editorial Prentice Hall Hispanoamericana, S.A., México.

6. Deming, W. Edwards. (1998). "Como Implementar el Kraizen en el sitio de trabajo (Gemba)". Editorial McGraw-Hill Interamericana, S.A., Bogota.

7. Imai, Masaaki. (1998). "Como implementar el Kraizen en el sitio de trabajo (Gemba)". Editorial McGraw-Hill Interamericana, S.A., Bogota.

8. Juran, José M. (1990). "Juran y la Planificación para la Calidad". Ediciones Díaz Santos, S.A., Madrid.

9. Heizer, Jay y Render, Barry. (1997). "Dirección de la Producción. Desiciones Estratégicas". Editorial Prentice Hall Hispanoamericana, S.A., Madrid.

10. Rincón, Haydee C. (2000). "Sistemas de Costos. Convencional y Actual". Publicación de la Facultad de Ciencias Económicas y Sociales. Universidad de los Andes, Mérida-Venezuela.

11. Ruelas, B., Enrique. (1993). "Calidad, Productividad y Costos", Revista de Salud Publica, Vol. 35 - № 3, México. 


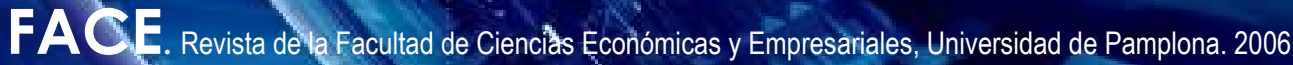

\section{PLANES DE NEGOCIO GANADORES}

\section{CONVOCATORIA FONDO EMPRENDER - SENA}

\author{
Proyecto: DISTRICAMPAM, \\ Distribuidora de productos campesinos de Pamplona \\ Emprendedores: Sonia Hernández \\ Franklin Giovanny Vargas \\ Estudiantes de Administración Comercial y de Sistemas \\ Universidad de Pamplona \\ Asesora Líder: Mary Luz Ordoñez Santos
}

\section{CONVOCATORIA FONDO EMPRENDER - SENA}

\section{Proyecto: INDUSTRIA DE CALZADO \\ Emprendedor: Ricardo Alfonso Maduro Mendoza \\ Estudiante de Ingeniería Industrial \\ Universidad de Pamplona \\ Asesora Líder: Laura Teresa Tuta Ramírez}

\section{Proyecto: CENTRO DE ESTETICA DE PAMPLONA}

Emprendedores: Diana Patricia Mora Ramón

Nadia Tamar González Rangel

Estudiantes de Administración de Empresas

Universidad de Pamplona

Asesor Líder: Luís Manuel Palomino Méndez

\section{Proyecto: MYOSCANNER}

Emprendedores: Lewis Andrés Ballesteros Mobil

Estudiante de Ingeniería Electrónica

Mathelen Ivonne Torres Vera

Estudiante de Administración de Empresas

Universidad de Pamplona

Asesor Líder: Heriberto Rangel

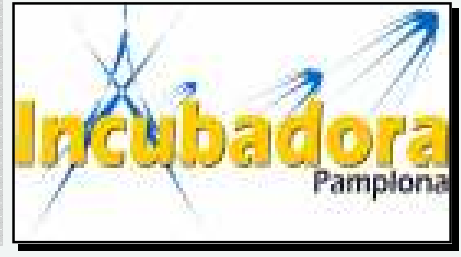




\section{Artículos Publicados Ediciones Anteriores}

Vol: 1\#1, Enero - Junio 2005

IMPACTO DE LA DEMANDA DE SERVICIOS, DE LA POBLACIÓN UNIVERSITARIA FORÁNEA, EN LA DINÁMICA ECONÓMICA DEL MUNICIPIO DE PAMPLONA.

Mary Luz Ordoñez Santos

Jorge Enrique Téllez Páez

Juan Manuel Villamizar Ramírez

Samuel Duarte Figueroa

Grupo de Investigación UNIR Universidad-Región

INCUBADORA DE EMPRESAS Y PLANES DE NEGOCIO. LA CULTURA DEL EMPRENDIMIENTO

Álvaro Parada Carvajal.

Grupo de Investigación COMPRENDER

LINEAMIENTOS DE INGENIERÍA DE SOFTWARE EDUCATIVO BASADOS EN MODELOS PEDAGÓGICOS, BAJO UNA METODOLOGÍA DE PROYECTOS PARA NIÑOS DE NIVEL PREESCOLAR

Ailin Orjuela Duarte

Mauricio Rojas Contreras

Grupo de Investigación CECOM

ESTRATEGIAS CULTURALES DE NEGOCIACIÓN: NEGOCIADORES FINLANDESES EN EL MERCADO COMERCIAL LATINOAMERICANO

Pâivi Johanna Vaahterikko-Mejia

Vol: 1 \#2, Junio - Diciembre 2005

RIESGO Y VOLATILIDAD DEL TIPO DE CAMBIO EN COLOMBIA.

Evaluación y Pronóstico 2000 - 2006

Mary Luz Ordoñez Santos

Grupo de Investigación UNIR Universidad-Región

ANALISIS DE COMPETITIVIDAD DEL MAÍZ AMARILLO DENTRO DE LA CADENA AVICOLA EN COLOMBIA 1999 - 2004

Antonio Martínez Reina

José de Jesús Montoya

LA EDUCACIÓN UNIVERSITARIA EN EL SIGLO XXI

DESCENTRALIZACIÓN Y DÉFICIT FISCAL EN COLOMBIA

Mary Luz Ordoñez Santos

Rigoberto Pedraza Pérez

Grupo de Investigación UNIR Universidad-Región

Indicaciones para los colaboradores: http://revistaface.unipamplona.edu.co Contacto: revistaface@unipamplona.edu.co 
FACE

CONGRESOS ORGANIZADOS POR LA FACULTAD DE CIENCIAS ECONOMICAS Y EMPRESARIALES.

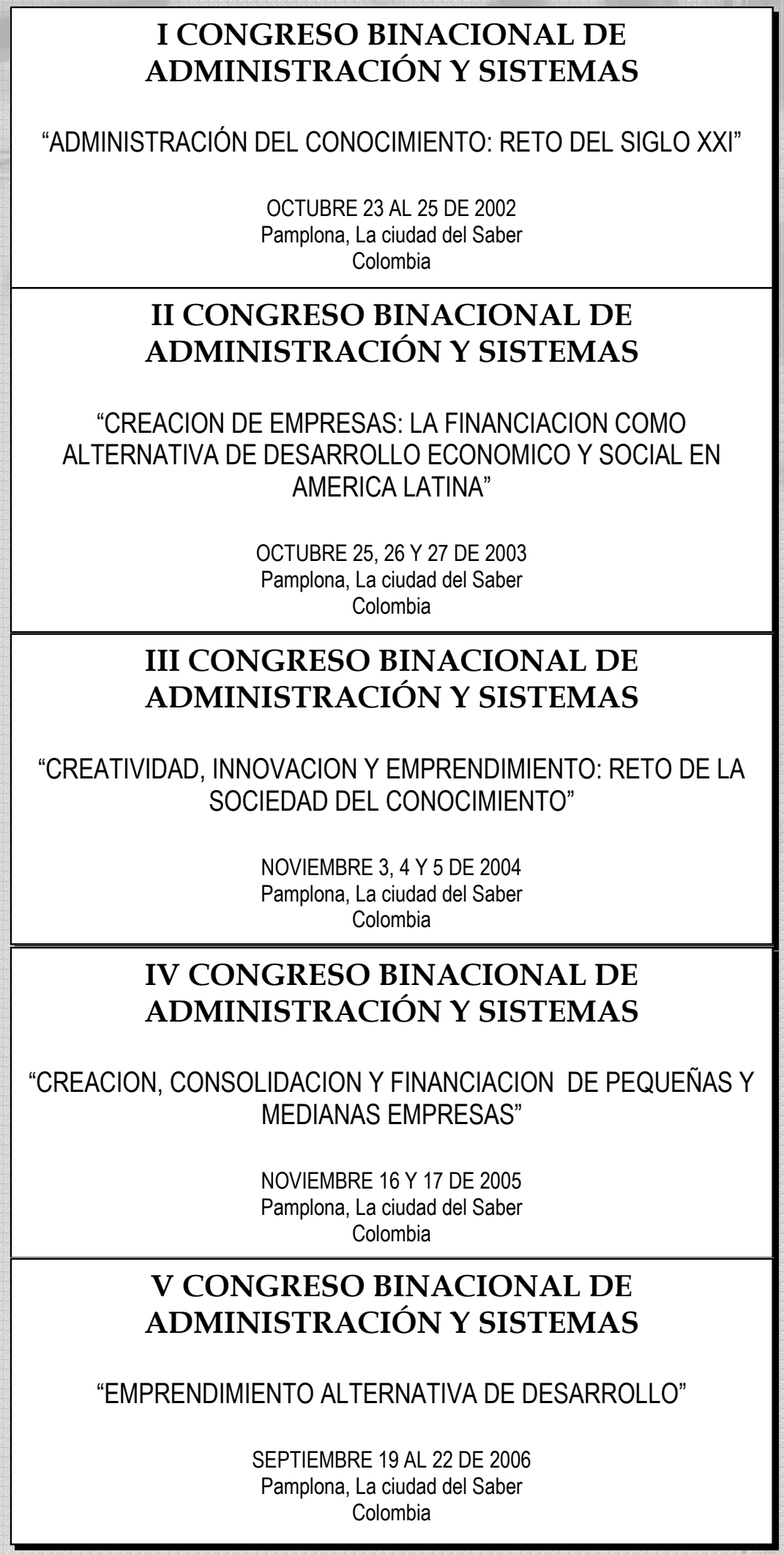

